Patterning photo-curable light-emitting organic composites by vertical and horizontal capillarity: a general route to photonic nanostructures

This article has been downloaded from IOPscience. Please scroll down to see the full text article.

2008 Nanotechnology 19335301

(http://iopscience.iop.org/0957-4484/19/33/335301)

View the table of contents for this issue, or go to the journal homepage for more

Download details:

IP Address: 137.108.145.45

The article was downloaded on 08/05/2013 at 06:10

Please note that terms and conditions apply. 


\title{
Patterning photo-curable light-emitting organic composites by vertical and horizontal capillarity: a general route to photonic nanostructures
}

\author{
Elisa Mele $^{1}$, Andrea Camposeo ${ }^{1}$, Carmela De Marco ${ }^{1,2}$, \\ Luana Persano $^{1}$, Roberto Cingolani ${ }^{1}$ and Dario Pisignano ${ }^{1,2}$ \\ ${ }^{1}$ NNL, National Nanotechnology Laboratory of CNR-INFM, Università del Salento, \\ via Arnesano, I-73100 Lecce, Italy \\ ${ }^{2}$ Scuola Superiore ISUFI, Università del Salento, via Arnesano, I-73100 Lecce, Italy \\ E-mail: dario.pisignano@unile.it
}

Received 9 April 2008, in final form 19 May 2008

Published 7 July 2008

Online at stacks.iop.org/Nano/19/335301

\begin{abstract}
We demonstrate the patterning of organic light-emitting composites made by conjugated polymers and photo-curable matrices. Using blends that exhibit both the structural properties of a low-viscosity photo-curable polymer, and the emission features of conjugated light-emitting materials, we study the operational principle of the pattern transfer and the modifications induced by the imprinting and microfluidic procedures on the optical properties of the compounds investigated. The combination of high-resolution silicone and perfluoropolyether elastomeric templates and low-viscosity, light-emitting polymeric blends offers the possibility to easily produce active organic structures with $100 \mathrm{~nm}$ scale resolution, thus extending current nanopatterning routes to this important class of composite material.
\end{abstract}

(Some figures in this article are in colour only in the electronic version)

\section{Introduction}

Patterning active organic molecules is nowadays a main issue of worldwide research, in view of the realization of flexible, low-cost plastic optoelectronic devices [1], such as photonic crystals (PhCs), distributed feedback structures, and organic light-emitting diodes (OLEDs). In particular, it is important to realize features characterized by one dimension (lateral or vertical, respectively) well below $1 \mu \mathrm{m}$. In fact, for slab PhCs the period of the imprinted gratings has to match the emission wavelength of the active material employed (400-600 nm), which results in a reduction of the self-absorption within the organic film and possibly in an increase in the amount of the output light $[2,3]$. For OLEDs, the thickness of the active layer has to be about $100 \mathrm{~nm}$, in order to have efficient electronhole recombination $[4,5]$. For these reasons, lithographic approaches able to guarantee a high control of the lateral and vertical size of the realized organic structures are required.
Hence, a lot of effort is being dedicated to developing novel lithographies, preserving the functionality of conjugated molecular compounds [6-9]. Soft step and flash lithography (SFL) [10] and microfluidic lithography ( $\mu$ FL) [11] are promising, simple processing methods to produce high-quality patterns of active molecules. The key elements of both SFL and $\mu \mathrm{FL}$ are a low-viscosity, photo-curable polymer, which allows one to carry out the imprint/molding process at room temperature, and a transparent template, ensuring the complete exposure of the photopolymer.

In conventional SFL [12, 13], the rigid transparent stamp is pressed onto the photopolymerizable liquid. The polymer fluid fills the void spaces between the template and the substrate, driven both by capillary force and by longrange polymer transport induced by the applied pressure. The performances of SFL can be further improved by the substitution of the rigid template with an elastomeric stamp or replica $[14,15]$. Such a combination of step and flash, and soft lithography offers some advantages in comparison to other 
imprint approaches [16, 17], especially for patterning organics. Elastomeric elements, such as poly(dimethylsiloxane) (PDMS) replicas, spontaneously wet surfaces by conformal contact over a large area (up to many $\mathrm{cm}^{2}$ ), without applying external pressures, and they are generally chemically inert, thus not sticking to the target material. Consequently, the mold surface treatments, required in the conventional nanoimprint lithography (NIL) and SFL for a complete release from the polymeric layer, can be avoided [18]. The duplication of the initial geometry in elastomeric copies prolongs the life of the original master, avoiding the stresses due to the direct use for imprinting.

Another approach for obtaining high-quality micro- and nano-features is the $\mu \mathrm{FL}$ technique [11]. In this case, the elastomeric stamp has to incorporate a network of hydraulically communicating capillaries, connected to external or on-chip reservoirs of liquids. A curable prepolymer is employed to make the channels spontaneously filled by capillary action. After curing, the pattern is stably transferred onto the substrate. In contrast to other approaches for texturing active organics, such as soft molding [19] and roomtemperature NIL $[8,20]$, soft SFL and $\mu$ FL perform direct patterning without applying high temperature and pressure.

Unfortunately, standard soft SFL and $\mu \mathrm{FL}$ can only be applied to a small range of low-viscosity photo-curable compounds. Photo-induced molding could only be applied on very few low-molar-mass molecules, and a previous report did not indicate the values of the luminescence efficiency after patterning [21]. In more recent experiments, a semiconductorlike functionality could be obtained by adding triphenylamine analogs within photo-curable monomers [22], or, vice versa, a photo-curable behavior was achieved by including oxetane sidegroups into electroluminescent polymers [23]. Using solution-processable composites including both the photocurable material and the light emitter would be advantageous in terms of cost and throughput.

In this work, we demonstrate the patterning of organic photoluminescent materials, using composites that exhibit both the structural properties of a low-viscosity photocurable polymer, and the emission of conjugated light-emitting compounds. We realized periodic patterns with resolution and aspect ratio varying over decades (from $5 \mu \mathrm{m}$ to $300 \mathrm{~nm}$ and from 0.8 to $7 \times 10^{-3}$, respectively) by soft SFL and $\mu$ FL. We investigated the changes in the optical properties of the active materials due to the realization of photonic microstructures, and compared the quantum efficiency of the untextured and the patterned film, observing an increment (from $2.4 \%$ to more than a factor 2) upon patterning.

\section{Experimental details}

\subsection{Materials}

Standard PDMS was purchased from Dow Corning (Sylgard 184, Midland, MI), and the solution was prepared by mixing Sylgard 184 base (A) and curing agent (B) in a ratio of 9 parts $\mathrm{A}$ and 1 part $\mathrm{B}$, by weight. The chemical compounds used for hard PDMS ( $h$-PDMS) solution are trimethylsiloxy-terminated vinylmethylsiloxanedimethylsiloxane (VDT-731), Pt catalyst (platinum divinyltetramethyldisiloxane, SIP6831.1), and methylhydrosiloxanedimethylsiloxane (HMS-301), each purchased from ABCR (Karlsruhe, Germany), and a modulator (2,4,6,8 tetramethyltetravinylcyclotetrasiloxane), purchased from Sigma Aldrich (St Louis, MO). The perfluoropolyether (PFPE) was kindly provided by Solvay Solexis. Polyurethane (NOA 72) was purchased from Norland Optical Adhesive (NJ). The lightemitting polymers, poly[\{2-methoxy-5-(2-ethylhexyloxy $\}-1,4-$ (1-cyanovinylenephenylene))-co- $\left\{2,5\right.$-bis $\left(\mathrm{N}, \mathrm{N}^{\prime}\right.$-diphenyl-amino)1,4-phenylene\}] (RE), poly[(9,9-dioctylfluorenyl-2,7-diyl)-co(1,4-diphenylene-vinylene-2-methoxy-5-\{2-ethylhexyloxy\}-benzene)] (GE), and poly[(9,9-hexylfluorenyl-2,7-ditl)-alt-co-(9ethyl-3,6-carbazole)] (BE), were purchased from American Dye Source, Inc. (Baie D. Urfe, Quebec, Canada).

\subsection{Procedures}

Master patterns (to be used in SFL) with parallel grooves of period $600 \mathrm{~nm}$ were fabricated on silicon by electron beam lithography and reactive ion etching. Other masters were made of $10 \mu \mathrm{m}$ squares and gratings with period between 10 and $30 \mu \mathrm{m}$, fabricated by photolithography on $\mathrm{Si} / \mathrm{SiO}_{2}$ (thickness $\cong 100 \mathrm{~nm}$ ) substrates.

The composite stamps of hard PDMS ( $h$-PDMS) and PDMS fabricated by the master were made up of a thin layer (30-40 $\mu \mathrm{m})$ of $h$-PDMS, bonded to a thick slab $(3 \mathrm{~mm})$ of PDMS. The $h$-PDMS solution was prepared mixing $3.4 \mathrm{~g}$ of VDT-731, $18 \mu \mathrm{l}$ of SIP6831.1, and $5 \mu \mathrm{l}$ of modulator. Afterward the mixture was degassed for 1-2 min, $1 \mathrm{~g}$ of HMS301 was added, and the $h$-PDMS solution was gently stirred. We then spin-coated (1500 rpm for $40 \mathrm{~s}$ ) a thin $h$-PDMS layer onto the master surface. After polymerization, PDMS was poured onto the $h$-PDMS layer and cured at $60^{\circ} \mathrm{C}$ for $1 \mathrm{~h}$. Then, the composite stamp was carefully peeled off from the master surface.

For the realization of PFPE-based molds, the prepolymer was obtained by mixing the PFPE monomer with $2 \% \mathrm{w} / \mathrm{w}$ of photoinitiator Darocur $^{\circledR} 1173$ (Ciba) (2-hydroxy-2-methyl-1phenyl-propan-1-one). After stirring, the prepolymer was spincoated on the master at $150 \mathrm{rpm}$ for $20 \mathrm{~s}$. Afterward the system was UV-cured under nitrogen atmosphere.

In the SFL procedure, the elastomeric replicas were placed onto a polymeric film spin-coated at $1500 \mathrm{rpm}$ for $40 \mathrm{~s}$ onto Corning glass substrate. The polymeric film was made up of NOA 72 and a light-emitting polymer in 20:1 w/w ratio. In order to obtain a homogeneous polymeric blend, the organic molecules were diluted in chloroform, and NOA 72 was added after the complete solvent evaporation. After positioning the replica, the system was exposed to UV light (45 W) through the backside of the template, under vacuum $\left(10^{-3} \mathrm{mbar}\right)$, for 10, 20, and $30 \mathrm{~h}$ for RE, GE, and BE composites, respectively. Afterward, the mold was separated from the substrate, and the pattern remained on the target.

For $\mu \mathrm{FL}$ experiments, the PFPE molds were placed in conformal contact with glass substrates, and with indium-tinoxide (ITO) coated glass, both bare, i.e. treated by an oxygen 
plasma (rf power of $200 \mathrm{~W}$ for $20 \mathrm{~min}$ ), and previously spuncast with a typically employed hole-transporting, $100 \mathrm{~nm}$ thick layer, namely poly(3,4-ethylene dioxythiophene) (PEDOT) doped with poly(styrenesulfonate) (PSS). The microfluidic networks were filled by the photo-curable active solution by capillary force, and exposed to UV light (as described for SFL).

\subsection{Instrumentation}

Fluorescence micrographs of the light-emitting patterns were collected with a Leica MZ16FA stereomicroscope coupled to a $3264 \times 2448$ camera, under excitation by a Hg lamp. For the measurement of the absolute quantum efficiency, we used as exciting source a Xe lamp matched with a monochromator $(\lambda=390 \mathrm{~nm})$, and we collected the PL signal in an integrating sphere, by a fiber-coupled charge-coupled device (Ocean Optics, FL) [24]. The number of PL photons per absorbed photon emitted by the untextured and the patterned films was determined by accounting for those photons which are not absorbed by the sample at their first incidence, and are absorbed after successive reflections on the surface of the integrating sphere: $\Phi_{\mathrm{PL}}=\frac{P_{1}-(1-\alpha)^{2}(R+T) P_{2}}{(1-R-T)(1-\alpha) X_{\mathrm{L}}}$, where $R$ and $T$ indicate the reflectance and transmittance of the system, respectively. $P_{1}$ and $P_{2}$ are the photoluminescence signals measured with the lamp beam incident on the sample and on the integrating sphere, respectively. $X_{\mathrm{L}}$ stands for the excitation signal, measured with the lamp incident on the sphere without the sample, and the factor $(1-\alpha)^{2}$ takes into account the correction due to the absorption of the glass substrate. All the signals were normalized by the spectral response of the experimental setup.

\section{Results and discussion}

In the case of SFL (figure 1(a)), the spontaneous capillarity flow direction of the photo-curable composite is along the $z$ axis (vertical capillarity), whereas for $\mu \mathrm{FL}$ (figure 1(b)) the polymer fills the capillaries along the $x$ axis (horizontal capillarity). First we fabricated master gratings with period between $600 \mathrm{~nm}$ (figure 2(a)) and $30 \mu \mathrm{m}$, and squares with lateral dimension of $10 \mu \mathrm{m}$. Standard PDMS often does not allow a faithful replication of features in the $500 \mathrm{~nm}$ range and with a very low aspect ratio, due to its low Young's modulus ( $3 \mathrm{MPa}$ ). For accurate generation of sub- $\mu \mathrm{m}$ patterns by SFL, we therefore fabricated composite elastomeric stamps, based on vinyl and hydrosilane end-linked polymers ( $h$-PDMS) [25], having higher Young's modulus (9 MPa) [26, 27]. Instead, for the realization of structures with lateral size of several $\mu \mathrm{m}$ and height of $100 \mathrm{~nm}$ by $\mu \mathrm{FL}$, neither PDMS nor $h$-PDMS are good candidates for the definition of microfluidic capillaries (having aspect ratio below $10^{-2}$ ), since the occurrence of collapse of the elastomeric structures interrupts the hydraulic connection with the fluid reservoirs. Hence, we realized the $\mu \mathrm{FL}$ molds by a PFPE polymer, which exhibits the advantages of PDMS in terms of UV transparency, flexibility, and conformal contact, moreover withdrawing the limits of collapse and swelling in organic solvents [28, 29].

We used the elastomeric molds to transfer the pattern of the master onto two-component polymeric films, made by (a)
SFL technique

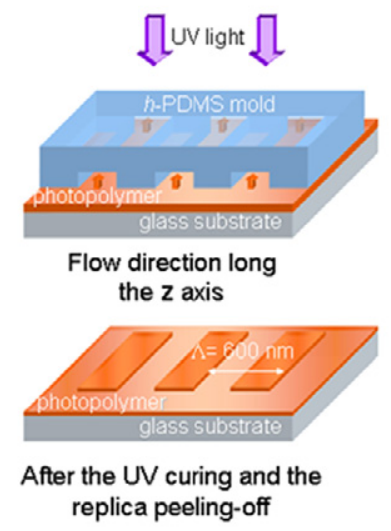

(b) $u F N$ technique

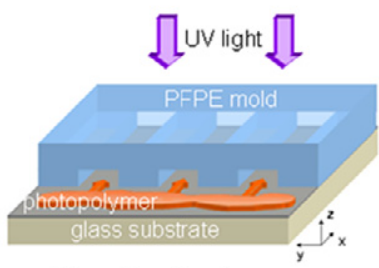

Flow direction long the $\mathrm{x}$ axis

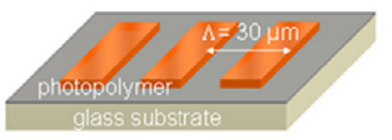

After the UV curing and the replica peeling-off
Figure 1. Schematic representation of the SFL (a) and the $\mu$ FL (b) processes (features not in scale).

a low-viscosity, photo-curing polyurethane (NOA 72), and a conjugated luminescent polymer. Several compounds were patterned, including a green-emitting (GE), a red-emitting (RE), and a blue-emitting (BE) polymer (molecular structures in figures 2(b)-(d)). During molding, the system was exposed to UV light through the backside of the template to prime the crosslinking, at room temperature and in a low-vacuum chamber $\left(10^{-3}\right.$ mbar). This allowed us to reduce the degradation of the optical properties of the active compounds, mainly induced by oxygen incorporation and substitution during the UV exposure [30].

We observe that the advantages of soft SFL and $\mu \mathrm{FL}$ for structuring light-emitting organics are given by the combined use of elastomeric, possibly solvent-resistant templates and of suitable polymeric composites. The low viscosity of the mixture, relying on the photo-curing material, enables the capillary penetration in the nanochannels constituted by the features of the mold. The embedded conjugated polymers determine the emission properties of the resulting nanostructure. In order to fully exploit the structural characteristics of the photo-curable compound, we optimized the relative weight fractions in the blend. A too low amount (i.e. comparable to or lower than that of the active polymer) of NOA72 can cause an incomplete filling of the nanochannels because of poor capillarity, whereas a too high fraction can significantly reduce the emission yield of the blend. A typical organic grating realized on the light-emitting blend is shown in figures 2(e) and (f). The comparison of the imprinted pattern with the master demonstrates the excellent fidelity achieved. In particular, the organic features present a lateral size of $300 \mathrm{~nm}$, and a depth of $(250 \pm 5) \mathrm{nm}$. No significant difference was observed between gratings fabricated by exposing the samples to UV light immediately after positioning the replica on the organic film and with a time delay up to tens of minutes after placing the mold. The well-defined grating indicates that the achieved resolution depends on the starting master, and that it can be further improved for reaching a sub-100 nm scale.

In the lithographic processes, the capillary force drives the organic composite into the recessed features formed between 
(a)

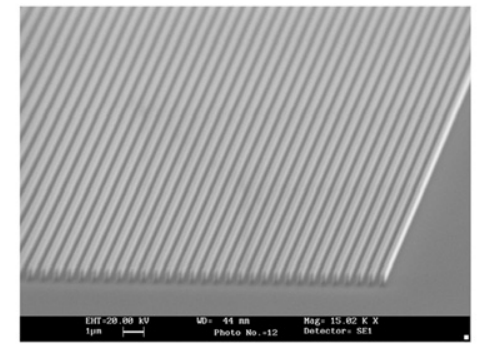

(b)
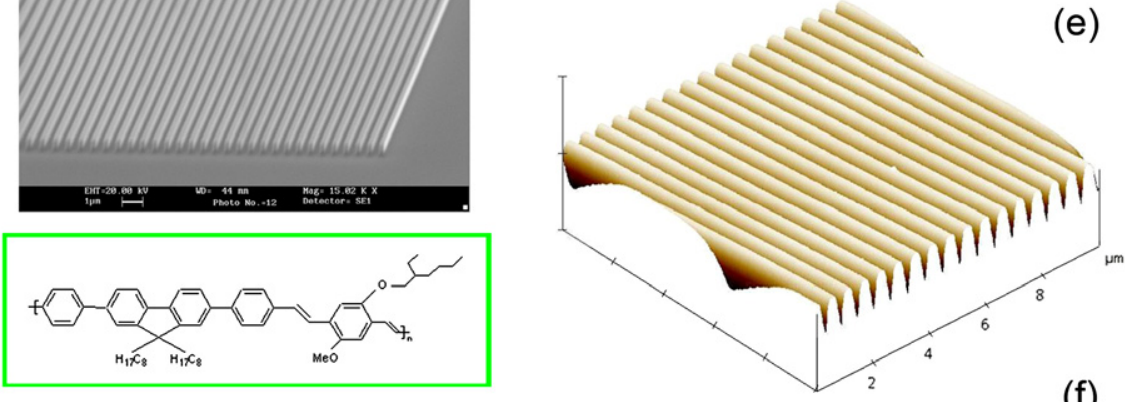

(c)
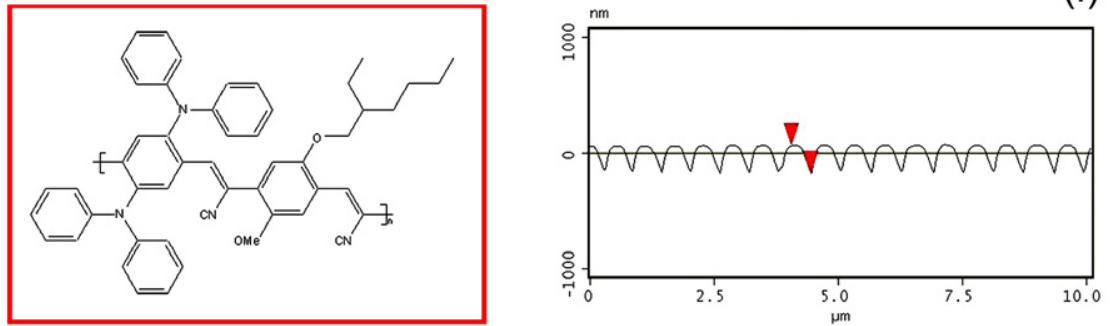

(d)

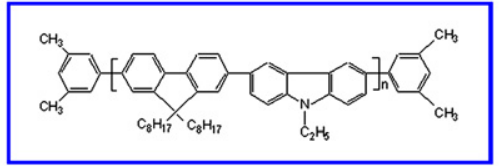

Figure 2. Three-dimensional view, imaged by scanning electron microscopy (SEM), of the master of the $600 \mathrm{~nm}$ period grating (a).

Molecular structures of the conjugated polymer employed: GE (b), RE (c), and BE (d). (e) Three-dimensional view, imaged by atomic force microscopy (AFM), and corresponding cross section of the imprinted $600 \mathrm{~nm}$ grating (f).

mold and substrate. In SFL, the filled height of the fluidic channels, with of vertical coordinate, $z$, generally depends on the penetration time, $t$, by the following relationship [31]:

$$
z=\left(\frac{R \gamma t \cos \Theta}{2 \eta_{\mathrm{s}}}\right)^{1 / 2},
$$

where $\eta_{\mathrm{s}}$ is the viscosity of the polymeric solution, $R$ is the hydraulic radius of the capillary, $\gamma$ is the surface tension at the solution/air interface, and $\Theta$ is the contact angle between the solution and the surface of the capillary. Equation (1) still remains valid in the case of $\mu \mathrm{FL}$ pattern transfer, the coordinate $z$ being replaced by the channel length, namely by the horizontal coordinate, $x$. The viscosity of the polymer mixture can be expressed by the equation [32]

$$
\ln \eta_{\mathrm{s}}=\sum_{i} w_{i} \ln \eta_{i}
$$

where $w_{i}$, and $\eta_{i}$ are the weight fraction and the viscosity of component $i$ in the blend, respectively. In the case of a binary solution of NOA $72(i=1)$ and a light-emitting polymer $(i=2)$, equation (2) becomes $\eta_{\mathrm{s}}=\eta_{1}^{w_{1}} \eta_{2}^{w_{2}}$. The rheological properties of the blend vary during the curing time. In particular, the viscosity of the photo-curing polyurethane changes during the process, whereas the viscosity of the active organic component remains constant. In general, the complex phenomenology of the curable polymer system makes difficult exactly determining the maximum height (or length) reachable by the blend before becoming solid, at least in the case of simultaneous curing and capillary filling.

In order to assess the possible influence of patterning on the emission properties of the conjugated polymers in the polyurethane matrix, we studied the light-emitting patterns and the photoluminescence (PL) spectra before and after the lithography process. A set of fabricated light-emitting structures incorporating the different conjugated materials employed is displayed in figures 3(a)-(e), evidencing welldefined and uniform features and, for $\mu \mathrm{FL}$ samples, the absence of unsought luminescent bottom layers. Furthermore, for all the molecules investigated, the integrated PL spectra are largely unchanged upon curing (figure 3(f)), showing small variations (both red- and blue-shifts, depending on the compound employed) in the peak wavelength, $\lambda_{\mathrm{PL}}$ (of less than 4\%). The composites also show a significant reduction in the linewidth (full width at half maximum, FWHM), $\Delta \lambda_{\mathrm{PL}}$, upon SFL (up to $24 \%$; see table 1). This may be attributed to variations in the molecular electronic environment and structural packing upon matrix polymerization. In particular, by high-resolution (wavelength-scale) patterning, the conjugated polymer/matrix blend undergoes two concomitant effects: the UV-induced polymerization of the embedding matrix, which can cause a deterioration of the emission properties of the light-emitting molecules, and the engraving of the periodic features by imprinting, which transfers a PhC-like structure on the organic slab, thus further affecting the optical properties. 
(a)

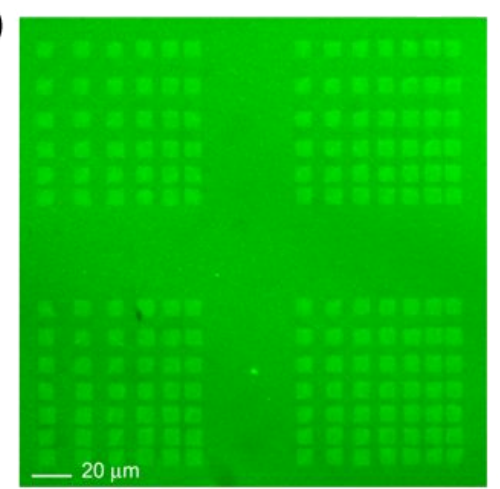

(c)

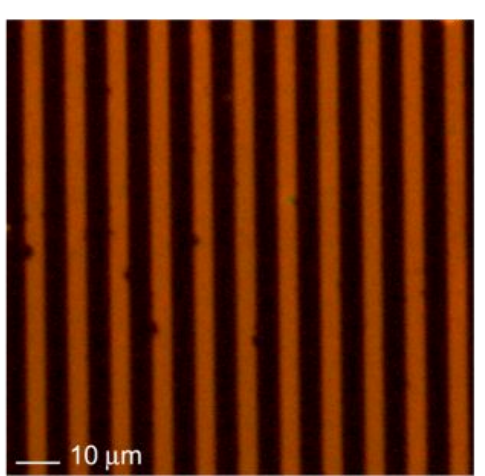

(e)

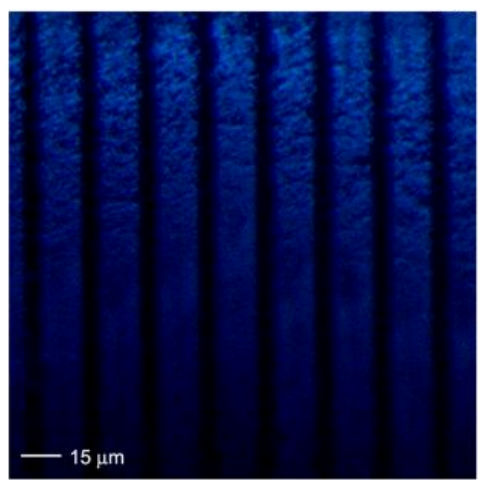

(b)

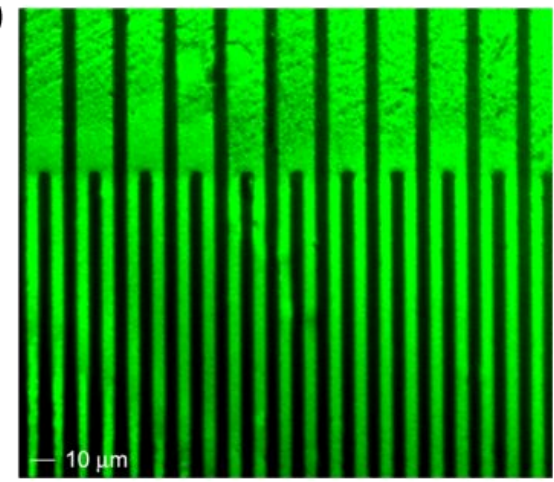

(d)

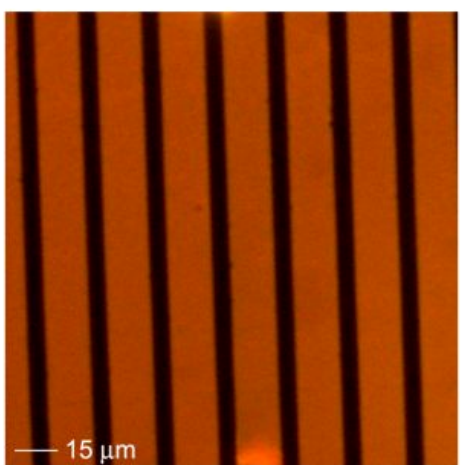

(f)

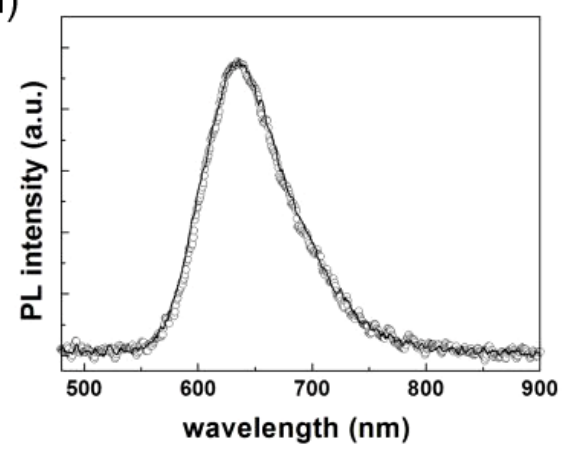

Figure 3. ((a)-(e)) Patterned microstructures of light-emitting composites incorporating GE ((a), (b)), RE ((c), (d)) and BE (e) conjugated polymers within different geometries. (f) PL spectra before (line) and after (circles) SFL patterning for a prototypical composite with RE polymer.

Table 1. Emission spectral properties, and PL quantum yields of the patterned blends, before and after the lithography process, and for the patterned and the unpatterned regions (grating period $600 \mathrm{~nm}$ ).

\begin{tabular}{|c|c|c|c|c|c|c|c|c|c|}
\hline \multirow[b]{2}{*}{ Compound } & \multicolumn{3}{|c|}{ Before polymerization } & \multicolumn{6}{|c|}{ After polymerization } \\
\hline & $\begin{array}{l}\lambda_{\mathrm{PL}} \\
(\mathrm{nm})\end{array}$ & $\begin{array}{l}\Delta \lambda_{\mathrm{PL}} \\
(\mathrm{nm})\end{array}$ & $\begin{array}{l}\Phi_{\mathrm{PL}} \\
(\%)\end{array}$ & $\begin{array}{l}\lambda_{\text {PL unpatterned }} \\
(\mathrm{nm})\end{array}$ & $\begin{array}{l}\Delta \lambda_{\mathrm{PL} \text { unpatterned }} \\
(\mathrm{nm})\end{array}$ & $\begin{array}{l}\Phi_{\text {PL unpatterned }} \\
(\%)\end{array}$ & $\begin{array}{l}\lambda_{\text {PL patterned }} \\
(\mathrm{nm})\end{array}$ & $\begin{array}{l}\Delta \lambda_{\text {PL patterned }} \\
(\mathrm{nm})\end{array}$ & $\begin{array}{l}\Phi_{\text {PL patterned }} \\
(\%)\end{array}$ \\
\hline RE & 637 & 86 & 27.5 & 646 & 80 & 24.4 & 635 & 83 & 25.0 \\
\hline GE & 518 & 56 & 67.0 & 517 & 46 & 21.1 & 515 & 53 & 23.0 \\
\hline $\mathrm{BE}$ & 446 & 81 & 20.3 & 426 & 61 & 4.7 & 426 & 54 & 10.2 \\
\hline
\end{tabular}

To investigate the influence of the lithographic procedure further, we measured the PL absolute quantum efficiency, $\Phi_{\mathrm{PL}}$, of the polymeric mixtures before and after SFL. Comparing the values of the PL yield after curing, for unpatterned and patterned regions, allowed us to discriminate the influence of the imprinted grating (with period $\Lambda=600 \mathrm{~nm}$ ) on the emission efficiency. In all the blends investigated, we found that patterned films exhibit a remarkable increment of $\Phi_{\mathrm{PL}}$, from $2.4 \%$ to more than a factor 2 . This improvement is comparable with the best results to date obtained on conjugated systems by non-photoexposure methods [8]. The increase of $\Phi_{\mathrm{PL}}$ is due to the insertion of the one-dimensional, wavelength- 
scale periodic nanostructure into the light-emitting films [2, 3]. In fact, the photon scattering process is governed by the conservation of the in-plane component of the wavevector of the output light, $\mathbf{k}_{\mathbf{0}}\left(\left|\mathbf{k}_{\mathbf{0}}\right|=\frac{2 \pi}{\lambda}\right)$, and of the guided mode $\mathbf{k}_{\mathbf{g}}$ $\left(\left|\mathbf{k}_{\mathbf{g}}\right|=\frac{2 \pi n_{\text {eff }}}{\lambda}\right)$ by the Bragg vector, $\mathbf{G}\left(|\mathbf{G}|=\frac{2 \pi}{\Lambda}\right): \mathbf{k}_{\mathbf{0}} \sin \Psi=$ $\pm \mathbf{k}_{\mathbf{g}} \pm n \mathbf{G}$, where $\Psi$ is the angle of emission, $n_{\text {eff }}$ is the effective refractive index of the waveguided mode, and $n$ is an integer. The light trapped in waveguide modes is Bragg scattered out of the film along the forward direction, thus reducing the selfabsorption within the organic slab and determining an increase of the external quantum efficiency. As a consequence of the reduced self-absorption in the high-energy tail of the emission spectra, we also noted a blue-shift of $\lambda_{P L}$ for some investigated compounds (table 1).

These methods allow one to concomitantly exploit the structural properties of the photo-curing polymer, and the emission properties of conjugated compounds. In principle, these composites can also provide effective paths for the current flow through the films; hence they can be employed as active layers for patterned electroluminescent devices. The matrices can be useful to diminish the energy transfer from a high-band-gap to a low-band-gap emitting species, thus allowing one to more effectively control the resulting color of the emitted light for certain active blends [33]. In particular, PFPE-assisted $\mu$ FL allows one to obtain a very good control on the resulting height of the organic microstructures, and to realize $100 \mathrm{~nm}$ thick active patterns, which is crucial for electron-hole recombination $[4,5]$, and very hard to be achieved by spin-coating with nanocomposite materials.

In conclusion, we applied soft SFL and $\mu \mathrm{FL}$ for structuring organic light-emitting composites, realizing onedimensional PhCs. We studied the operational principle of the pattern transfer and the optical properties of the organic compounds investigated. The techniques described, combining the use of high-resolution elastomeric templates and lowviscosity, light-emitting polymeric blends, offer the possibility to easily produce active organic structures with $100 \mathrm{~nm}$ scale resolution. These extend the use of soft lithography to a novel, important class of composite material, thus being promising candidates for plastic nanofabrication.

\section{Acknowledgments}

This work was partially supported by the Apulia Regional Strategic Project 'Ponamat' and by the Italian Minister of University and Research through the FIRB project RBIP06SH3W. We gratefully thank Dr A Zompatori, SolvaySolexis for the PFPE elastomer. We also thank F Di Benedetto for the AFM investigation.

\section{References}

[1] Menard E, Meitl M A, Sun Y, Park J-U, Jay-Lee Shir D, Nam Y-S, Jeon S and Rogers J A 2007 Chem. Rev. $1071117-60$

[2] Lupton J M, Matterson B J, Samuel I D W, Jory M J and Barnes W L 2000 Appl. Phys Lett. 77 3340-2
[3] Matterson B J, Lupton J M, Safonov A F, Salt M G, Barnes W L and Samuel I D W 2001 Adv. Mater. 13 123-7

[4] Friend R H et al 1999 Nature 397 121-8

[5] Zaumseil J, Friend R H and Sirringhaus H 2006 Nat. Mater. 5 69-74

[6] Holdcroft S 2001 Adv. Mater. 13 1753-65

[7] Wang J, Sun X, Chen L and Chou S Y 1999 Appl. Phys. Lett. 75 2767-9

[8] Pisignano D, Persano L, Raganato M F, Visconti P, Cingolani R, Barbarella G, Favaretto L and Gigli G 2004 Adv. Mater. 16 525-9

[9] Boroumand F A, Fry P W and Lidzey D G 2005 Nano Lett. 6 67-71

[10] Mele E, Di Benedetto F, Persano L, Pisignano D and Cingolani R 2005 Nanotechnology 16 391-5

[11] Kim E, Xia Y and Whitesides G M 1996 J. Am. Chem. Soc. 118 5722-31

[12] Colburn M et al 1999 Proc. SPIE: Emerging Lithographic Technologies 3676 379-89

[13] Colburn M, Grot A, Amistoso M, Choi B J, Bailey T, Ekerdt J, Sreenivasan S V, Hollenhorst J and Willson C G 2000 Proc. SPIE: Emerging Lithographic Technologies 3997 453-7

[14] Xia Y and Whitesides G M 1998 Angew. Chem. Int. Edn 37 550-75

[15] Xia Y, Kim E, Zhao X-M, Rogers J A, Prentiss M and Whitesides G M 1996 Science 273 347-9

[16] Chou S Y, Krauss P R and Renstrom P J 1995 Appl. Phys. Lett. $673114-6$

[17] Chou S Y, Krauss P R and Renstrom P J 1996 Science 272 85-7

[18] Bailey T, Choi B J, Colburn M, Meissl M, Shaya S, Ekerdt J G, Sreenivasan S V and Wilson C G $2000 \mathrm{~J}$. Vac. Sci. Technol. B $183572-7$

[19] Pisignano D, Persano L, Cingolani R, Gigli G, Babudri F, Farinola G M and Naso F 2004 Appl. Phys. Lett. 84 1365-7

[20] Pisignano D, Persano L, Visconti P, Cingolani R, Gigli G, Barbarella G and Favaretto L 2003 Appl. Phys. Lett. $832545-7$

[21] Rogers J A, Meier M, Dodabalapur A, Laskowski E J and Cappuzzo M A 1999 Appl. Phys. Lett. 74 3257-9

[22] Behl M, Seekamp J, Zankovych S, Sotomayor Torres C M, Zentel R and Ahopelto J 2002 Adv. Mater. 14 588-91

[23] Müller C D, Falcou A, Reckefuss N, Rojahn M, Wiederhirn V, Rudati P, Frohne H, Nuyken O, Becker $\mathrm{H}$ and Meerholz K 2003 Nature 421 829-33

[24] Greenham N C, Samuel I D W, Hayes G R, Phyllips R T, Kessener Y A R R, Moratti S C, Holmes A B and Friend R H 1995 Chem. Phys. Lett. 241 89-96

[25] Schmid H and Michel B 2000 Macromolecules 33 3042-9

[26] Odom T W, Love J C, Wolfe D B, Paul K E and Whitesides G M 2002 Langmuir 18 5314-20

[27] Odom T W, Thalladi V R, Love J C and Whitesides G M 2002 J. Am. Chem. Soc. 124 12112-3

[28] Rolland J P, Hagberg E C, Denison G M, Carter K R and De Simone J M 2004 Angew. Chem. Int. Edn 43 5796-9

[29] Rolland J P, Van Dam R M, Schorzman D A, Quake S R and DeSimone J M 2004 J. Am. Chem. Soc. 126 2322-3

[30] Yan M, Rothberg L J, Papadimitrakopoulos F, Galvin M E and Miller T M 1994 Phys. Rev. Lett. 73 744-7

[31] Meyers D 1999 Surface, Interface, and Colloids (New York: Wiley-VCH) pp 97-118

[32] Song Y, Mathias P M, Tremblay D and Chen C-C 2003 Ind. Eng. Chem. Res. 42 2415-22

[33] Granström M and Inganäs O 1996 Appl. Phys. Lett. 68 147-9 